\title{
Unintentional Discontinuation of Statins May Increase Mortality After Traumatic Brain Injury in Elderly Patients: A Preliminary Observation
}

\author{
Alessandro Orlando ${ }^{\mathrm{a}, \mathrm{b}}$, David Bar-Ora, b, c, g, Kristin Salottolo ${ }^{\mathrm{a}, \mathrm{b}}$, Andrew Stewart Levy ${ }^{\mathrm{d}}$, \\ Charles W Mains, ${ }^{\mathrm{a}, \mathrm{c}, \mathrm{e}}$, Denetta S Slone ${ }^{\mathrm{f}}$, Patrick J Offner ${ }^{\mathrm{a}, \mathrm{e}}$
}

\begin{abstract}
Background: The abrupt discontinuation of statin therapy has been suggested as being deleterious to patient outcomes. Although pre-injury statin (PIS) therapy has been shown to have a protective effect in elderly trauma patients, no study has examined how this population is affected by its abrupt discontinuation. This study examined the effects of in-hospital statin discontinuation on patient outcomes in elderly traumatic brain injury (TBI) patients.
\end{abstract}

Methods: This was a multicenter, retrospective cohort study on consecutively admitted elderly ( $\geq 55$ ) PIS patients who were diagnosed with a blunt TBI and who had a hospital length of stay (LOS) $\geq 3$ days. Patients who received an in-hospital statin within 48 hours of admission were considered continued, and patients who never received an in-hospital statin were considered discontinued. Differences in in-hospital mortality, having at least one complication, and LOS $>1$ week were examined between those who continued and discontinued PIS.

Results: Of 93 PIS patients, 46 continued and 15 discontinued statin therapy. The two groups were equivalent vis-a-vis demographic and clinical characteristics. Those who discontinued statin therapy had a 4-fold higher mortality rate than those who continued $(\mathrm{n}=4,27 \%$ vs. $\mathrm{n}=3,7 \%, \mathrm{P}=0.055)$. Statin discontinuation did not

\footnotetext{
Manuscript accepted for publication March 21, 2013

${ }^{\mathrm{a} T r a u m a}$ Research Department, St. Anthony Hospital, Lakewood, CO, USA

${ }^{\mathrm{b}}$ Trauma Research Department, Swedish Medical Center, Englewood, CO, USA

${ }^{\mathrm{c}}$ College of Osteopathic Medicine, Rocky Vista University, Parker, CO, USA

${ }^{\mathrm{d}}$ InterMountain Neurosurgery, St. Anthony Hospital, Lakewood, CO, USA

${ }^{\mathrm{e}}$ Trauma Services Department, St. Anthony Hospital, Lakewood, CO, USA

${ }^{\mathrm{f}}$ Trauma Services Department, Swedish Medical Center, Englewood, CO, USA

${ }^{\mathrm{g}}$ Corresponding author: David Bar-Or, Trauma Research Department, 501 E. Hampden Ave, Room 4-454, Englewood, CO 80113, USA.

Email: dbaror@ampiopharma.com
}

doi: http://dx.doi.org/10.4021/jocmr1333w have a higher complication rate, compared to statin continuation (n $=3,20 \%$ vs. $\mathrm{n}=7,15 \%, \mathrm{P}=0.70)$, and no difference was seen in the proportion with a hospital LOS $>1$ week $(\mathrm{P}>0.99)$.

Conclusions: Though our study is not definitive, it does suggest that the abrupt, unintended discontinuation of statin therapy is associated with increased mortality in the elderly TBI population. Continuing in-hospital statin therapy in PIS users may be an important factor in the prevention of in-hospital mortality in this elderly TBI population.

Keywords: Statin; Discontinuation; Traumatic brain injury; Trauma; Elderly

\section{Introduction}

The majority of statin consumers are over the age of 50 [1] and simultaneously have a high risk of suffering a traumatic brain injury (TBI) [2]. In elderly trauma patients, pre-injury statin (PIS) therapy has been shown to have a protective effect [3-5]; nevertheless, the discontinuation of PIS therapy upon hospital admission has been recently suggested as having deleterious effects on patient outcomes. These studies examined cardiac $[6,7]$, stroke $[8,9]$ and vascular surgery populations [10], yet the elderly TBI population remains unexamined. The reasons for in-hospital statin discontinuation can be intentional, e.g. the patient is too severely injured to receive oral medications, or unintentional, e.g. clinical oversight. The objective of this study was to examine the extent to which unintentional statin discontinuation increases poor outcomes in the elderly TBI population. We hypothesized that unintentional statin discontinuation would be associated with worse patient outcomes, compared to continuation.

\section{Methods}

\section{Study site and patient population}

This study is a retrospective review of consecutively ad- 


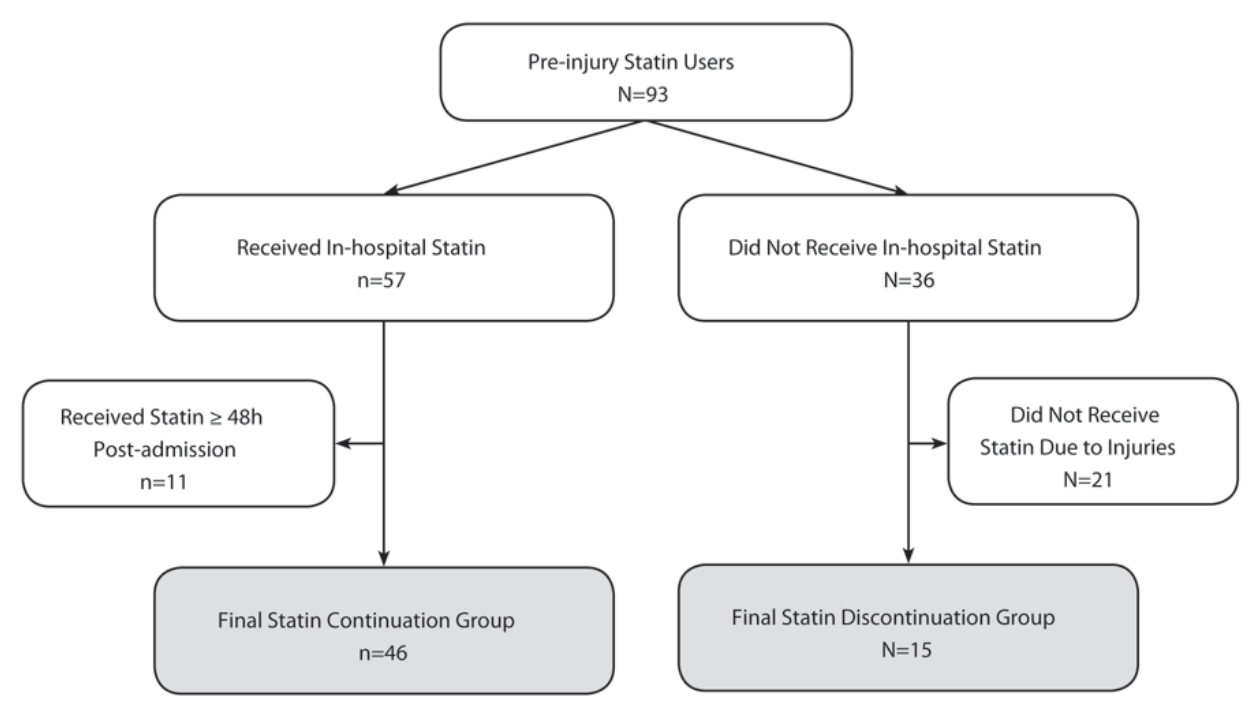

Figure 1. Study population flow-chart.

mitted adult trauma patients to two Level I Trauma Centers (first center: May 1, 2007 - November 20, 2008; second center: September 1, 2007 - August 31, 2009) with a blunt TBI which included at least one of the following diagnoses: cerebral contusion, subarachnoid hemorrhage or other unspecified intracranial hemorrhage, subdural or epidural hematoma, or diffuse axonal injury. Patients were excluded from the study because of no PIS use, age $<55$ years, and a hospital length of stay (LOS) less than three days. This study was reviewed and approved by each institution's Institutional Review Board.

Pre-injury statin use was identified as a statin medication in a patient's paper chart or electronic medical record. If a PIS patient was never administered an in-hospital statin, they were considered to be discontinued from statin therapy. If a PIS patient's in-hospital medications included the administration of a statin, they were considered to be continued on statin therapy.

It is likely that severe injuries prevent some patients from being continued on an in-hospital statin. Therefore, independent, qualified hospital physicians and nurses reviewed the medical charts of all patients who were discontinued. $\mathrm{Pa}$ tients who were found to be intentionally discontinued from statin therapies within 48 hours of hospital admission for injury-related reasons (e.g. care withdrawal or intubation) were excluded to avoid bias (Fig. 1). Only those patients who were discontinued due to clinical oversight were included in the discontinuation group. There was no difference between hospitals in the rate of discontinuation due to clinical oversight $(P=0.74)$. Furthermore, we ensured that the cause of death was not related to a failure to comply with preventative care protocols (for example, deep vein thrombosis prophylaxis). If a do not resuscitate (DNR) protocol was activated in a patient with a likely survivable condition, that patient was excluded from the study.

\section{Outcomes and covariates}

The primary outcome of this study was in-hospital mortality. Secondary outcomes were having at least one in-hospital complication, and a hospital LOS $>1$ week. Covariates were defined as follows: age (years 55 - 79, $\geq 80$ ), ED Glasgow Coma Scale (GCS, 3 - 12, 13 - 15), injury severity score (ISS, $0-15, \geq 16$ ), ED hypotension (systolic blood pressure $<90$ $\mathrm{mmHg}$ ), ED respiratory rate (abnormal ( $<10$ or $>29$ breaths/ minute), normal (10 - 29)), ED tachycardia ( $>100$ beats per minute), body mass index (BMI, normal (18.5 - $24.9 \mathrm{~kg} / \mathrm{m}^{2}$ ) and underweight (16.5 - 18.4), vs. overweight (25 - 29.9) and obese $(\geq 30)$ ), mechanism of injury (fall, motor-vehicle accident and other), gender, pre-injury blood thinner use (clopidogrel, aggrenox, aspirin or warfarin), blood product transfusion (including fresh frozen plasma, packed red blood cells and albumin), Trauma Injury Severity Scale (TRISS) and the Charlson Comorbidity Index (CCI).

\section{Statistical analysis}

Significant changes in inflammatory markers [11-15], and increased associations with mortality $[7,9]$ have been reported within 24 - 72 hours of statin therapy discontinuation. We chose 48 hours as the cutoff time within which patients had to have their in-hospital statin therapy continued. Based on the literature, we felt 48 hours was the average time after which we should expect to see statin discontinuation effects. Thus, we compared outcomes between patients who discontinued statin therapy upon hospital admission and those who 
Table 1. Patient Demographics Stratified by In-Hospital Statin Therapy Continuationa

\begin{tabular}{|c|c|c|c|}
\hline Characteristics & $\begin{array}{l}\text { Continued } \\
(n=46)\end{array}$ & $\begin{array}{l}\text { Discontinued } \\
(\mathrm{n}=15)\end{array}$ & P Value \\
\hline Age & & & 0.75 \\
\hline 55 to 79 & $30(65 \%)$ & $11(73 \%)$ & \\
\hline$\geq 80$ & $16(35 \%)$ & $4(27 \%)$ & \\
\hline Male & $25(53 \%)$ & $10(67 \%)$ & 0.40 \\
\hline BMI overweight or obese $\left(\geq 25 \mathrm{~kg} / \mathrm{m}^{2}\right)$ & $29(67 \%)$ & $4(29 \%)$ & 0.01 \\
\hline Pre-injury blood thinner use & $40(87 \%)$ & $10(67 \%)$ & 0.12 \\
\hline ED GCS $13-15$ & $37(93 \%)$ & $12(92 \%)$ & $>0.99$ \\
\hline ISS $0-15$ & $16(35 \%)$ & $5(33 \%)$ & 0.92 \\
\hline ED hypotension $(<90$ mmHg) & $1(2 \%)$ & 0 & $\mathrm{n} / \mathrm{a}$ \\
\hline ED respiratory rate (breaths/min) & & & 0.42 \\
\hline$<10$ or $>29$ & $1(2 \%)$ & $1(7 \%)$ & \\
\hline 10 to 29 & $44(98 \%)$ & $13(93 \%)$ & \\
\hline ED tachycardia (> 100 bpm) & $7(16 \%)$ & $3(21 \%)$ & 0.69 \\
\hline TRISS, probability of survival $\geq 80 \%$ & $35(92 \%)$ & $12(92 \%)$ & $>0.99$ \\
\hline Charlson comorbidity index & & & 0.32 \\
\hline 2 to 3 & $7(15 \%)$ & $4(27 \%)$ & \\
\hline 4 to 6 & $30(64 \%)$ & $10(67 \%)$ & \\
\hline$\geq 7$ & $10(21 \%)$ & $1(7 \%)$ & \\
\hline Mechanism of Injury & & & 0.18 \\
\hline Fall & $37(80 \%)$ & $12(80 \%)$ & \\
\hline Motor vehicle or bicycle & $8(17 \%)$ & $1(7 \%)$ & \\
\hline Other & $1(2 \%)$ & $2(13 \%)$ & \\
\hline Blood product transfusion & $11(23 \%)$ & $3(20 \%)$ & 0.72 \\
\hline ED ECG results & & & $>0.99$ \\
\hline Abnormal & $27(75 \%)$ & $10(77 \%)$ & \\
\hline Borderline & $4(11 \%)$ & $1(8 \%)$ & \\
\hline Normal & $5(14 \%)$ & $2(15 \%)$ & \\
\hline ED disposition & & & 0.70 \\
\hline Direct admission & $4(9 \%)$ & $1(7 \%)$ & \\
\hline General floor & $9(20 \%)$ & $2(13 \%)$ & \\
\hline Intensive care unit & $31(67 \%)$ & $10(67 \%)$ & \\
\hline Step-down unit & $2(4 \%)$ & $2(13 \%)$ & \\
\hline
\end{tabular}

aNot all data will sum to their respective totals due to missing data. BMI: body mass index; ED: emergency department; GCS: glasgow coma scale; ISS: injury severity score; BPM: beats per minute; TRISS: trauma and injury severity score; ECG: electrocardiogram. 


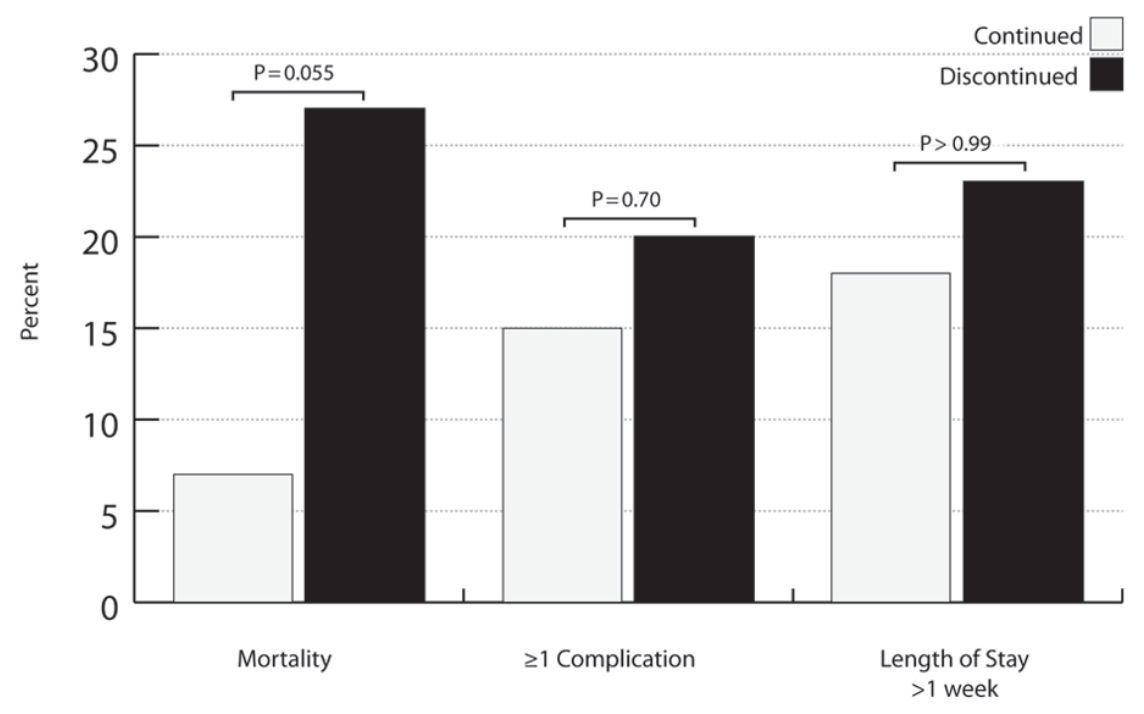

Figure 2. Outcomes between pre-injury statin continuation and discontinuation groups.

continued statin therapy within 48 hours of hospital admission.

Pearson or Fisher's exact chi-square tests were used to examine the effects of statin discontinuation on in-hospital mortality, the development of any complications and having a hospital LOS $>1$ week. Only patients who survived to hospital discharge were included in the LOS analysis. All statistical analyses were performed using $\mathrm{SAS}{ }^{\circledR}$ software, version 9.1 (SAS Institute, Cary, NC), and had an alpha value of 0.05 .

\section{Results}

A total of 93 patients were on PIS therapy, and the statin discontinuation rate was $38 \%(\mathrm{n}=36)$. After exclusion criteria were applied, the final study population consisted of 61 patients; 46 statin continuation and 15 statin discontinuation patients (Fig. 1). The most commonly prescribed in-hospital statin therapy was simvastatin $(\mathrm{n}=27,59 \%)$, followed by atorvastatin $(\mathrm{n}=12,26 \%)$, lovastatin $(\mathrm{n}=5,11 \%)$, pravastatin $(\mathrm{n}=1,2 \%)$ and rosuvastatin $(\mathrm{n}=1,2 \%)$. Both statin continuation and discontinuation groups were predominately male, had normal vital signs upon admission, mild head injuries, high ISS and TRISS survival probabilities, and similar CCI scores (Table 1). Body mass index was the only significant demographic difference between study groups; the statin continuation group had a significantly higher proportion of overweight or obese patients $(\mathrm{P}=0.01)$.

The overall mortality rate was $11 \%(\mathrm{n}=7)$. The statin discontinuation group had a mortality rate 4 -fold higher than the statin continuation group, trending toward statistical significance $(\mathrm{n}=4,27 \%$ vs. $\mathrm{n}=3,7 \%, \mathrm{P}=0.055)$. Due to the small sample size, we did not adjust for any variables; though, there were no significant demographic or clinical characteristics between study groups, except BMI $(\mathrm{P}=0.01)$. The chart review revealed that 6 of the 7 deaths had an attached DNR; however, all DNRs were activated because of very poor expected outcomes and unlikely recoveries. Furthermore, no patient died from a failure to comply with a preventative care protocol.

The complication rates were similar between the two study groups $(\mathrm{n}=3,20 \%$ vs. $\mathrm{n}=7,15 \%, \mathrm{P}=0.70)$. In the discontinuation group, there were six complications across the three patients, and in the discontinuation group, there were 18 complications across the seven patients. The majority of complications were infection-related in both study groups (continuation: 37\%; discontinuation: 67\%). There was no significant difference in the proportion of patients with a LOS $>1$ week between statin discontinuation and continuation groups $(\mathrm{n}=3,18 \%$ vs. $\mathrm{n}=11,23 \%, \mathrm{P}>0.99)$.

\section{Discussion}

Physicians have long known the dangers associated with the sudden discontinuation of beta blockers, and much like beta blockers, the sudden discontinuation of statin therapy has also been suggested as being detrimental to patients' health [6-10, 16-18]. Our study presents preliminary data that are in agreement with this theory and expands the affected population to include elderly TBI patients. Patients in our study who had unintended discontinuation of PIS had a mortality rate that trended towards being statistically significantly higher than those patients who were continued within 48 hours of admission (Fig. 2) $(\mathrm{P}=0.055)$. 
Two large studies examining statin therapy continuation have demonstrated that statin discontinuation is associated with higher mortality rates. Spencer et al. studied PIS use in over 19,500 patients across 14 countries with acute coronary syndrome [7]. They found that patients who had their statin therapy discontinued had a significantly higher mortality rate compared to those who continued $(12 \%$ vs. $2 \%, \mathrm{P}<0.001)$. In another study, Spencer et al. examined a subset of patients from the National Registry of Myocardial Infarction and investigated the effects of PIS use in over 78,000 patients with myocardial infarction [6]. After adjustment, discontinuation of statin therapy was associated with a significant increase in the risk of mortality compared to patients who were continued ( $\mathrm{HR}=1.83,95 \% \mathrm{CI}: 1.58$ to 2.13$)$. Neither of these large-scale studies discounted the possibility that injury severity was associated with discontinuation and contributed to the increased mortality rate.

Meldrum and Moore et al. have presented compelling evidence suggesting that an initial insult, such a traumatic injury, primes the immune system for response, and a second event within a vulnerable time period may stimulate systemic inflammation $[19,20]$. This two-event construct may explain why statin discontinuation, versus continuation, trended towards having a significantly increased mortality rates in our study and in other patient populations $[6,8,9,16$, 17]. In our study, the TBI can be considered the first event, and the discontinuation of statin therapy might have acted as the second event. This theory is further reinforced by studies documenting changes in inflammatory markers following statin discontinuation, including decreased nitric oxide production, increased C-reactive protein and vascular cell adhesion molecule-1 levels $[11,12,15]$.

Necessary steps must be taken to identify and separate patients who were discontinued from statin therapy because of their injuries, from patients who were discontinued because of clinical oversight. Patients who are discontinued from statin therapy because their care has been withdrawn are expected to have a higher risk of mortality than patients who are capable of receiving an oral statin medication but were not prescribed one. Thus, the discontinuation of statin therapy can be thought of as an indication of the severity of a patient's injuries, and if not removed, will bias all outcome analyses [21]. Our study is unique in that it differentiated these discontinuation groups through a thorough chart review, ensuring that all instances of discontinuation were not related to events or indications that are associated with an increased mortality rate.

A full chart review might be impractical in large studies; however, the following variables would be beneficial in determining whether the severity of injury prevented the continuation of in-hospital statin therapy: nil per os, care withdrawal, DNR activation, receiving palliative care, intubation, coma, and emergent intervention. Furthermore, it is important to establish the temporality of the aforementioned variables. While some complications may prevent a patient from receiving in-hospital statins if they occur immediately after admission, other complications may be a consequence of not receiving an in-hospital statin. More importantly, the literature is void of a substantiated definition of statin withdrawal. Some studies illustrate deleterious effects after 24, 48 and 72 hours of statin discontinuation; our study chose a cut off value of 48 hours to define statin withdrawal. Future studies should aim to better understanding cellular and physiological changes subsequent to discontinuation.

The main limitation of our study was also one of its strengths: though our study had a small sample size, it made a thorough chart-review pragmatic. This allowed us to eliminate the possibility that statin therapy was discontinued because of a patient's injuries, and to ensure that all DNRs were activated in patients who had deteriorated beyond recovery. However, the small sample size limited our ability to adjust for possible confounding factors, and resulted in our study being underpowered to detect the differences we observed.

We examined our data for variables that might introduce bias. In Table 1 , we found three variables with a $\mathrm{P}<0.20$ : BMI, pre-injury blood thinner use and mechanism of injury. However, when we examined the associations between these variables and mortality, we discovered that none of them had a $\mathrm{P}<0.20$; therefore, these variables are not likely to severely confound our mortality analysis. Generalizability is limited because our two Level I Trauma Centers' standards of practice might differ from other hospitals. Despite our study's limitations, the demographic and clinical similarities between study groups, combined with the exclusion of patients who were discontinued from statins because of severe injuries, lead us to believe that our univariate analyses were not highly influenced by differences between study groups.

\section{Conclusions}

Though our study is not definitive, it does suggest that the abrupt, unintended discontinuation of statin therapy is associated with increased mortality in the elderly TBI population. The US Census Bureau estimates that the population aged 55 years and older will grow by 21 million over the next two decades [22]. It is likely that this population will continue to be a large consumer of statin medications, therefore our understanding of these patients and common medication usage must grow accordingly. Ensuring that a patient's PIS use is communicated between the ED physicians and nurses, and those in the admitting hospital unit might be a simple, yet important factor in the prevention of elderly TBI mortality.

\section{Acknowledgement}

Thank you to Michael Sullivan, Matt Vandam and Emily Gosche for chart abstraction. We would also like to thank 
the following individuals for conducting the chart-reviews: Anita Leyden, RN BSN; Pamela Bourg, RN MS; Glenda G Quan, MD.

\section{Declarations}

All authors state that they have no conflicts of interest to disclose.

\section{References}

1. Spatz ES, Canavan ME, Desai MM. From here to JUPITER: identifying new patients for statin therapy using data from the 1999-2004 National Health and Nutrition Examination Survey. Circ Cardiovasc Qual Outcomes. 2009;2(1):41-48.

2. Faul M, Xu L, Wald M, Coronado V. Traumatic brain injury in the United States: emergency department visits, hospitalizations, and deaths. Centers for Disease Control and Prevention, National Center for Injury Prevention and Control. 2010. Atlanta, GA.

3. Efron DT, Sorock G, Haut ER, Chang D, Schneider E, Mackenzie E, Cornwell EE, 3rd, et al. Preinjury statin use is associated with improved in-hospital survival in elderly trauma patients. J Trauma. 2008;64(1):66-73; discussion 73-64.

4. Schneider EB, Efron DT, MacKenzie EJ, Rivara FP, Nathens AB, Jurkovich GJ. Premorbid statin use is associated with improved survival and functional outcomes in older head-injured individuals. J Trauma. 2011;71(4):815-819.

5. Fogerty MD, Efron D, Morandi A, Guy JS, Abumrad NN, Barbul A. Effect of preinjury statin use on mortality and septic shock in elderly burn patients. J Trauma. 2010;69(1):99-103.

6. Spencer FA, Allegrone J, Goldberg RJ, Gore JM, Fox KA, Granger CB, Mehta RH, et al. Association of statin therapy with outcomes of acute coronary syndromes: the GRACE study. Ann Intern Med. 2004;140(11):857-866.

7. Spencer FA, Fonarow GC, Frederick PD, Wright RS, Every N, Goldberg RJ, Gore JM, et al. Early withdrawal of statin therapy in patients with non-ST-segment elevation myocardial infarction: national registry of myocardial infarction. Arch Intern Med. 2004;164(19):21622168.

8. Colivicchi F, Bassi A, Santini M, Caltagirone C. Discontinuation of statin therapy and clinical outcome after ischemic stroke. Stroke. 2007;38(10):2652-2657.

9. Blanco M, Nombela F, Castellanos M, Rodriguez-Yanez M, Garcia-Gil M, Leira R, Lizasoain I, et al. Statin treatment withdrawal in ischemic stroke: a controlled ran- domized study. Neurology. 2007;69(9):904-910.

10. Schouten O, Hoeks SE, Welten GM, Davignon J, Kastelein JJ, Vidakovic R, Feringa HH, et al. Effect of statin withdrawal on frequency of cardiac events after vascular surgery. Am J Cardiol. 2007;100(2):316-320.

11. Lee KT, Lai WT, Chu CS, Tsai LY, Yen HW, Voon WC, Sheu SH. Effect of withdrawal of statin on C-reactive protein. Cardiology. 2004;102(3):166-170.

12. Lai WT, Lee KT, Chu CS, Voon WC, Yen HW, Tsai LY, Sheu SH. Influence of withdrawal of statin treatment on proinflammatory response and fibrinolytic activity in humans: an effect independent on cholesterol elevation. Int J Cardiol. 2005;98(3):459-464.

13. Li JJ, Li YS, Chu JM, Zhang CY, Wang Y, Huang Y, Chen J, et al. Changes of plasma inflammatory markers after withdrawal of statin therapy in patients with hyperlipidemia. Clin Chim Acta. 2006;366(1-2):269-273.

14. Laufs U, Endres M, Custodis F, Gertz K, Nickenig G, Liao JK, Bohm M. Suppression of endothelial nitric oxide production after withdrawal of statin treatment is mediated by negative feedback regulation of rho GTPase gene transcription. Circulation. 2000;102(25):31043110.

15. Xing Y, Chen H, Hu DY. [Effects of withdrawal of statins on nitric oxide production in vascular endothelial cells]. Zhonghua Nei Ke Za Zhi. 2005;44(1):22-24.

16. Daskalopoulou SS, Delaney JA, Filion KB, Brophy JM, Mayo NE, Suissa S. Discontinuation of statin therapy following an acute myocardial infarction: a populationbased study. Eur Heart J. 2008;29(17):2083-2091.

17. Heeschen C, Hamm CW, Laufs U, Snapinn S, Bohm M, White HD. Withdrawal of statins increases event rates in patients with acute coronary syndromes. Circulation. 2002;105(12):1446-1452.

18. Risselada R, Straatman H, van Kooten F, Dippel DW, van der Lugt A, Niessen WJ, Firouzian A, et al. Withdrawal of statins and risk of subarachnoid hemorrhage. Stroke. 2009;40(8):2887-2892.

19. Meldrum DR, Cleveland JC, Jr., Moore EE, Partrick DA, Banerjee A, Harken AH. Adaptive and maladaptive mechanisms of cellular priming. Ann Surg. 1997;226(5):587-598.

20. Moore EE, Moore FA, Harken AH, Johnson JL, Ciesla D, Banerjee A. The two-event construct of postinjury multiple organ failure. Shock. 2005;24(Suppl 1):71-74.

21. Neal MD, Cushieri J, Rosengart MR, Alarcon LH, Moore EE, Maier RV, Minei JP, et al. Preinjury statin use is associated with a higher risk of multiple organ failure after injury: a propensity score adjusted analysis. J Trauma. 2009;67(3):476-482; discussion 482-474.

22. Projections of the Population by Age and Sex for the United States: 2010 to 2050. U.S. Census Bureau PD. 2008. Atlanta, GA. 